\title{
LONGITUDINAL TWO-YEAR FOLLOW-UP OF UPDATING AND FLEXIBILITY FUNCTIONS IN DRIVERS WITH PARKINSON'S DISEASE: PRELIMINARY RESULTS
}

\author{
Maud Ranchet ${ }^{1,2}$, Laurence Paire-Ficout ${ }^{1,2}$, \& Emmanuel Broussolle Em $^{3,4}$ \\ ${ }^{1}$ French Institute of Science and Technology for Transport, Development and Networks \\ (IFSTTAR - INRETS), LESCOT \\ ${ }^{2}$ Université de Lyon \\ ${ }^{3}$ Université Lyon I; Hospices Civils de Lyon, Hôpital Neurologique Pierre Wertheimer \\ ${ }^{4}$ Centre de Neurosciences Cognitives (CNRS) \\ Lyon, France \\ E-mail: maud.ranchet@ifsttar.fr
}

\begin{abstract}
Summary: This paper outlines the preliminary results of a longitudinal follow up at two years interval ( $\mathrm{t} 0$ versus $\mathrm{t} 2$ ) in 10 drivers with mild to moderate Parkinson's disease (PD) and 10 matched controls. Changes of (1) driving habits assessed by a questionnaire, (2) neuropsychological performances measured by a set of cognitive tests and (3) cognitive abilities while driving using a simulator, were analyzed. Two types of changes were observed: the decline over time in PD group at two years interval and the appearance of some deficits in $\mathrm{PD}$ patients (compared to controls) at the second assessment (t2). These deficits were not observed at the first one (t0). The results showed that PD patients had changed their driving habits over time (reduction of mileage, more avoidance, underestimation of their own driving competency). The cognitive status of PD patients remained relatively stable over time, except for the Trail Making Test performances (part A and part B) which declined. A deficit for the TMT-part A in PD patients, compared to controls, appeared at t2. The data from driving simulator showed no significant decline in PD patients for both updating and flexibility performances. However, a deficit in flexibility appeared at t2 in PD patients, as demonstrated by their poorer performances on the flexibility cost. Our data suggest that flexibility may be significantly affected in PD patients with more advanced disease. The small size on our sample does not allow us any conclusion on updating function in both PD patients and controls.
\end{abstract}

\section{INTRODUCTION}

Cognitive deficits may appear to be the main contributors to decreased driving performance in Parkinson's disease (PD) (Uc et al., 2006). Few studies have longitudinally investigated cognitive changes in PD patients. Some researchers observed a significant cognitive decline over eight years and longer (Brown et al., 1990). However, cognitive decline is slower over shorter periods of time. Some authors studied neuropsychological changes in PD patients approximately two years apart (Azuma et al., 2003). Results showed that executive functions are those most likely to be impaired by the disease progression. Deficits are also described in lower level executive functions such as mental flexibility (Cools et al., 2001), inhibition of automatic responses and the manipulation and updating of verbal and visuospatial representations (Postle et al., 1997). These functions are all particularly important in novel or demanding situations such as driving. In a recent paper, using neuropsychological and driving simulator tests, we showed 
impaired updating in PD drivers while mental flexibility remained unaffected (Ranchet et al., 2010). This dissociation could be due to the fact that patients were at relatively early stages of the disease. In addition, the cognitive status did not appear to be significantly impaired compared with controls. The purpose of the present study is to describe the preliminary results of a longitudinal follow up of cognitive performances in PD drivers. The same protocol, as the one used in our recent study, was applied in a subset of PD patients and controls two years after the first examination, focusing on the updating and flexibility functions. We also report the results of a questionnaire about driving habits filled out two years apart. Our hypothesis is that there should be a significant decline of PD group performances. Two types of changes have been observed (1) the decline over time in PD group at two years interval and (2) the appearance of some deficits in $\mathrm{PD}$ patients (compared to controls) at t2, not observed at the first one.

\section{METHODS}

\section{Population}

For this preliminary study, ten of the 25 PD patients and 10 of the 25 healthy controls studied in our first report (Ranchet et al, 2010) were included and re-tested approximately 26 months (t2) after the initial assessment (t0). The inclusion of participants at $\mathrm{t} 2$ is still on-going. Patients were matched to controls for age, education level and driving experience (table 1).

Table 1. Characteristics of PD Patients and Controls at T2

\begin{tabular}{lccc}
\hline & $\begin{array}{c}\text { PD Patients } \\
\mathrm{n}=10 \\
\text { mean (SD) }\end{array}$ & $\begin{array}{c}\text { Controls } \\
\mathrm{n}=10 \\
\text { mean (SD) }\end{array}$ & $\begin{array}{c}\text { Two-Tailed } \\
\mathrm{p} \text { - value }\end{array}$ \\
\hline Sex ratio (Men/ Women) & $8 / 2$ & $9 / 1$ & 0.579 \\
Age (y) & $68.0(4.2)$ & $69.1(4.7)$ & 0.002 \\
Mini Mental State Examination Score & $27.5(1.4)$ & $29.6(0.7)$ & 0.937 \\
Years of education & $13.9(3.6)$ & $14.6(3.2)$ & 0.631 \\
Years of driving & $48.6(5.0)$ & $50.0(7.7)$ & - \\
Disease onset age in years & $60.4(5.5)$ & - & - \\
Disease duration in years & $7.6(4.7)$ & - & - \\
Hoehn and Yahr stage (max: 5) & $2.4(0.3)$ & & \\
Unified PD Rating Scale - motor score (on medication & $18.6(4.1)$ & & - \\
$\quad$ max: 108) & $480.0(145.7)$ & & - \\
Levodopa dosage (mg/day) & & & \\
\hline
\end{tabular}

The inclusion criteria at $\mathrm{t} 2$ were the same than those applied at $\mathrm{t} 0$. All subjects held valid driving licences and they were regular drivers at the first assessment (with a minimum annual driving mileage of 1864 miles/year (3000km/year)). Note that the severity of Parkinson's has changed significantly over time. Indeed, patients were at stages more severe of the disease at t 2 than t0 (t2: $2.4 \pm 0.3$ vs t0: $1.80 \pm 0.35, \mathrm{p}=0.022$ ). They had also a significantly higher motor score at $\mathrm{t} 2$ compared to t0 (t2: $18.6 \pm 4.1$ vs t0: $16.2 \pm 5.8, \mathrm{p}=0.0001)$. They were still driving at the second assessment. The study was approved by the local biomedical ethics committee. Written informed consent was obtained from all subjects in accordance with Helsinki guidelines. 


\section{Questionnaire}

Participants were asked to fill out a 4-item questionnaire on perception of their own driving ability at both test times, selected from the literature. It focused on subject habits and difficulties while driving. Question $\mathrm{N}^{\circ} 1$ (asked at t0 and t2) was: "Currently, how many kilometers do you travel per year?” The participant had to choose among four answers (1. less than 1864 miles (less than 3000km); 2.between 1864 and 4350 miles (between 3500 and $7000 \mathrm{~km}$ ); 3.between 4350 and 12427 miles (between 7000 and $20000 \mathrm{~km}$ ); 4.more than 12427 miles (more than 20000 $\mathrm{km}$ ). Question $\mathrm{N}^{\circ} 2$ (asked at t0 and t2) was: “How do you judge your driving skills?” The participant had to give a mark score between 0 and 10. Question $\mathrm{N}^{\circ} 3$ (asked only at $\mathrm{t} 2$ ) was: "Which situations are more difficult than two years ago?” The participant had to tick all situations judged more difficult among seventeen propositions (highway driving, night driving etc...). The total number of difficult situations represented the difficulty score (on 17). Question $\mathrm{N}^{\circ} 4$ (asked only at t2) was: "Which driving situations do you avoid?” The same situations as in question $\mathrm{N}^{\circ} 3$ were proposed. The total number of avoided situations represented the avoidance score (on 17).

\section{Neuropsychological assessments}

Measures of global executive function were obtained using the Trail Making Test (TMT (B-A)). Ability to inhibit an automatic response, visual memory, and short-term storage capacity were assessed via the Stroop test (inhibition cost index), Benton Visual Retention Test, and a digit span task, respectively. Maintenance of relevant information (phonological loop), was measured using Baddeley's Working Memory digit-span task in single condition, in which participants were required to recall digit sequences of the same length for two minutes. The updating function was assessed via the n-back task with the 2-back condition as meaningful measure. Mental flexibility was assessed with the plus-minus task with the shift cost as the relevant measure.

\section{Driving simulator and experimental tasks}

Two scenarios were used to assess updating and flexibility functions while driving as previously described in a recent paper (Ranchet et al., 2010). In each situation, participants drove on a road with little traffic and a speed limit of 56 miles $/ \mathrm{h}(90 \mathrm{~km} / \mathrm{h})$.

Updating task. Participants were asked to recall, in any order, the last three road signs of each series while driving (free recall). They subsequently had to answer different questions about information given on the last three road signs (cued recall). Three series of four, six or eight road signs respectively varied randomly. The updating score, calculated by adding the free recall and cued recall scores, represented the updating measure.

Flexibility task. There were two different conditions. Firstly, in the condition without alternation, participants had to state the shape of the road sign (rectangular, triangular, square or round) placed on the right-hand side of the road (session 1). They then had to state the dominant colour of the road sign (blue, green, red or brown) placed on the left-hand side of the road (session 2). Each session included 3 practice trials and 16 tests. Secondly, in the condition with alternation (session 3, requiring mental flexibility), participants were asked to state the shape of the road 
sign if it was on the right-hand side, and to indicate the colour of the road sign if it was on the left. Signs were placed alternately on the right or left of the road. The condition with alternation included 4 practice trials and 32 tests. Flexibility cost constituted the mental flexibility measure; this was obtained by calculating the difference between mean reaction times of correct trials in the condition with alternation and mean reaction times of correct trials from the first two sessions in which no flexibility was required.

\section{Data analysis}

For the questionnaire data, sign tests were performed in each group to determine the number of patients who had significantly declined over time. Between-group and within-group differences regarding questionnaire, neuropsychological and driving simulator data were analyzed using non-parametric tests, depending on the variable normality (Shapiro-Wilk W Test, $p<.05$ ). Twotailed Wilcoxon rank-sum test was used to assess the statistical significance of changes in test performances over time. Two-tailed Mann-Whitney U test was used to compare differences between the two groups regarding questions, neuropsychological and driving simulator tests. An alpha level of 0.05 was applied to all our statistical analyses. All analyses were performed with SPSS 17.0 statistical software.

\section{RESULTS}

\section{Questionnaire Data}

A high number of patients declared to have restricted their mileage compared to those who remained stable ( $\mathrm{p}=0.037)$ : 6 patients versus 1 control reported to drive less kilometers at 2 compared to t0. Four patients versus 9 controls declared to drive as much as before. At t0, the judgment about their own driving competency did not differ between patients and controls (patients: mean $(M)=7.6 / 10 ; S D=1.3$ vs. controls: $M=7.8 / 10 \mathrm{SD}=1.3, \mathrm{p}=0.853$ ), whereas at $\mathrm{t}$, a significant difference in PD patients compared to controls on driving competences was observed (patients: mean=6.9/10; $\mathrm{SD}=1.1$ vs. controls: $\mathrm{M}=8.1 / 10 ; \mathrm{SD}=1.0, \mathrm{p}=0.025$ ). At t2, PD patients declared a higher number of difficult driving situations compared to controls (patients: $\mathrm{M}=4.4 / 17$; $\mathrm{SD}=3.6$ vs. controls: $\mathrm{M}=7.8 / 17$; $\mathrm{SD}=1.7, \mathrm{p}=0.035$ ). Similarly, they reported to avoid more driving situations than controls (patients: $M=5.5 / 17 ; \mathrm{SD}=3.0$ vs. controls: $\mathrm{M}=1.8 / 17 ; \mathrm{SD}=1.5, \mathrm{p}=0.006)$.

\section{Neuropsychological performances}

Global cognitive assessment. Among tests used to assess global cognitive performance, the PD group showed two significant declines over time in TMT-part A and TMT-part B: patients took significantly more time to perform both part A and part B of TMT at t2 compared to t0 (TMTpart A: $\mathrm{p}=0.04$; TMT-part B: $\mathrm{p}=0.04$ ). A significant difference between the two groups in TMTpart A appeared at t2; patients performed worse than the controls.

Updating: n-back task (Figure 1-A). In the patient group, no decline was recorded in 0-back, 1back and 2-back conditions. Interestingly, the decline over time in response times of the 2-back 
condition only appeared in control group $(\mathrm{p}=0.05)$ (Figure 1-A). No significant between-group differences in reaction times appeared at $\mathrm{t} 2$ in the n-back task.

Flexibility, plus-minus task (Figure 1-B). A decline tended to appear in patient group for the shift cost: a higher shift cost in patients was observed at t2 compared to t0 $(\mathrm{p}=0.09)$.

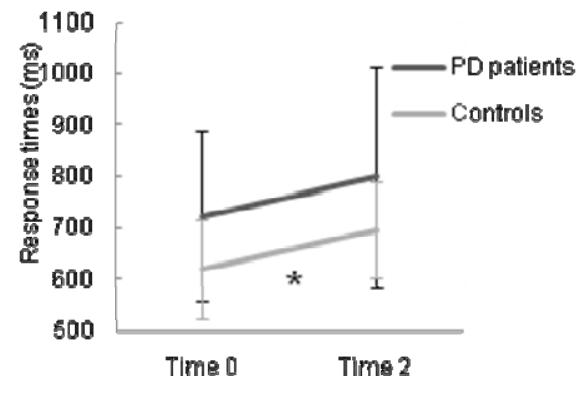

Figure 1-A. Changes over time in reaction times of 2-back condition in PD patients compared to controls $(*$ significant at $\mathrm{p}<0.05)$

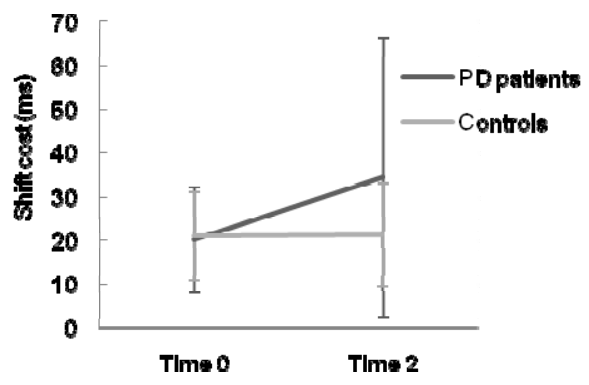

Figure 1-B. Changes over time in shift cost of plus-minus task in PD patients compared to controls

\section{Performances on driving simulator tasks}

Updating task (Figure 2-A). No significant differences in updating score were observed over time for both patients and controls, or between the two groups at t2. However, the variability (measured by SD) tended to increase between the two assessments particularly in the PD patients.

Flexibility task (Figure 2-B). Although no decline over time was observed in the flexibility cost, a significant difference between the two groups appeared at t2; patients had significantly higher flexibility cost than controls ( $\mathrm{p}=0.05$ ). Contrary to what we expected, all participants answered more quickly in both conditions with and without alternation at $\mathrm{t} 2$ compared to t0; however, this effect did not reach significance, (controls: $\mathrm{p}=0.07$; patients: $\mathrm{p}=0.14$ ).

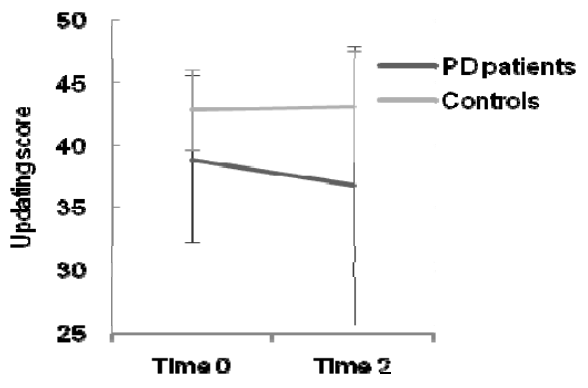

Figure 2-A. Changes over time in updating score in PD patients compared to controls on the driving simulator

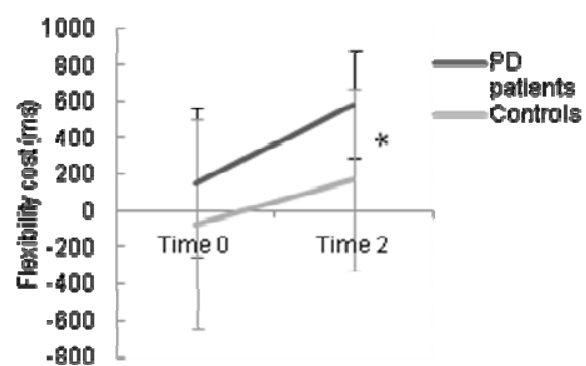

Figure 2-B. Changes over time in flexibility cost in PD patients compared to controls on the driving simulator $(*$ significant at $\mathrm{p}<0.05$ )

\section{DISCUSSION}

The present study investigated changes in driving habits, neuropsychological tests and driving simulator tasks in drivers with PD compared to controls, tested at approximately 2-year intervals. 


\section{Questionnaire on self-perception of driving ability}

The results of the questionnaire suggest that drivers with PD tend to modify their driving, even after two years of disease. Indeed, a large proportion self regulates their driving habits, reducing their number of miles driven per year and avoiding more driving situations. These results are in accordance with those described in the literature. Initially, there was no significant difference between the two groups on the self assessment of their driving skills. With the evolution of the disease, the perception of driving skills appears to be decreasing. Those in the PD group rated their driving skills more poorly compared to controls. These results suggest that patients are aware that their symptoms are affecting their driving performance (Klimkeit et al., 2009). Other analyses should be conducted in a larger sample size to confirm changes perceived over time.

\section{Global cognitive performances}

At first sight, our data show that the cognitive decline over two years is slow since most of performances from neuropsychological tests did not differ between the two assessments. However, we observed a significant decline over time in reaction times of TMT-part A in PD patients. Patient's performances were also consistently poorer than controls at t2. The TMT-part A performance, which measures basic information processing and psychomotor speed, appears to be sensitive to disease progression (Lezak, 1995). The TMT (B-A), considered in our study as a composite measure of global executive function, independently of psychomotor speed, did not significantly change between the two assessments in patients. Also, this score was not significantly different to those of controls at t2. These results suggest that there is no decline in global executive function in our PD group. This is consistent with previous reports showing relatively circumscribed executive dysfunction in PD (Azuma et al., 2003). Beyond these findings, one of the main objectives of this study was to investigate specific functions as updating and flexibility, in which we expected significant declines over time in the patient group.

\section{Updating and flexibility tasks}

Updating. Our recent study (Ranchet et al., 2010) revealed an updating impairment in our PD group at t0.This impairment was not observed at t2. Only one meaningful change over time was observed in 2-back condition in controls, suggesting a decline in updating ability with the normal ageing progression. In the updating task on simulator, there were no changes over time in both groups and no significant differences appeared at $\mathrm{t} 2$. A limited power due to the small sample size might have explained the absence of difference between groups.

Flexibility. At the first assessment, we showed that flexibility remained unaffected by Parkinson's disease (Ranchet et al., 2010). At the second assessment, patients had higher flexibility cost than controls for the driving simulator task, not observed in the plus-minus task. In our experiment on simulator, participants had to perform the flexibility task at the same time as driving, inducing an increase of cognitive load compared to neuropsychological tests. In accordance with some authors (Cools et al., 2001), this result reveals that the flexibility deficit is more pronounced when the load of attentional resources increases. Furthermore, these findings suggest that the flexibility function seems to be more affected in patient group at the second assessment even if their performances over time did not significantly decline. 
Limitations of our preliminary study. Generally, the absence of significant changes between the two assessments in patients could be due to higher heterogeneity in progression of Parkinson's disease compared to normal ageing (Tröster et al., 2007). A larger sample size would have given us more power to detect significant differences. Furthermore, we noticed that performances remained relatively stable or improved on driving simulator in both groups compared to neuropsychological tests. These findings could be due to practice effects. Indeed, with relatively difficult or novel tasks, people are apt to improve due to increased familiarity with the procedures (Lezak, 1995). Further supplementary analyses will be performed to confirm this hypothesis. In this study, the driving performances were not analyzed; other research (ongoing) will also demonstrate the effect of PD progression on driving performances.

\section{Conclusions}

This preliminary study indicates that flexibility appears to be more and more affected by the PD progression whereas updating function seems to remain stable over two years of disease. Further analyses on complete data (17 patients and 21 controls) will allow confirmation of these preliminary results and predictors of worsening flexibility cost in a larger sample of PD patients.

\section{AKNOWLEDGEMENTS}

We would like to thank Dr Claude Marin-Lamellet and Dr. Virginie Etienne for their initial contribution to this study. Many thanks to Marie France Boyer for the inclusion of all participants and Max Duraz for dealing with technical aspects of the driving simulator.

\section{REFERENCES}

Azuma, T., Cruz, R., Bayles, K. A., Tomoeda, C. K., \& Montgomery, E. B. (2003). A longitudinal study of neuropsychological change in individuals with Parkinson's disease. International Journal of Geriatric Psychiatry, 18(11), 1043-1049.

Brown, R. G., \& Marsden, C. D. (1990). Cognitive function in Parkinson's disease: from description to theory. Trends in Neurosciences, 13, 21-29.

Cools, R., Barker, R. A., Sahakian, B. J., \& Robbins, T. W. (2001). Mechanisms of cognitive set flexibility in Parkinson's disease. Brain, 124, 2503-2512.

Klimkeit, E. I., Bradshaw, J. L., Charlton, J., Stolwyk, R., \& Georgiou-Karistianis, N. (2009). Driving ability in Parkinson's disease: current status of research. Neuroscience \& Biobehavioral Reviews, 33(3), 223-231.

Lezak, M. D. (1995). Neuropsychological Assessment (3rd ed.). New York: Oxford University Press.

Postle, B. R., Locascio, J. J., Corkin, S., \& Growdon, J. H. (1997). The time course of spatial and object learning in Parkinson's disease. Neuropsychologia, 35, 1413-1422.

Ranchet, M., Paire-Ficout, L., Marin-Lamellet, C., Laurent, B., \& Broussolle, E. (2010). Impaired updating ability in drivers with Parkinson's disease. Journal Neurology Neurosurgery Psychiatry. 
Tröster, A. I., Woods, S. P., \& Morgan, E. E. (2007). Assessing cognitive change in Parkinson's disease: Development of practice effect-corrected reliable change indices. Archives of Clinical Neuropsychology, 22, 711-718.

Uc, E. Y., Rizzo, M., Anderson, S. N., Sparks, J. D., Rodnitzky, R. L., \& Dawson, J. D. (2006). Driving with distraction in Parkinson disease. Neurology, 67(2), 1774-1780. 